\title{
ON THE QUALITY OF CFOSAT SCATTEROMETER WINDS
}

\author{
Wenming Lin ${ }^{1}$, Marcos Portabella ${ }^{2}$, Shuyan Lang ${ }^{3}$, Xiaolong Dong ${ }^{4}$, Xingou Xu ${ }^{4}$, Zhixiong Wang ${ }^{1}$, Yijun \\ $H e^{l}$
}

${ }^{1}$ School of Marine Sciences, Nanjing University of Information Science and Technology, 210044, Nanjing, China
${ }^{2}$ Institute of Marine Sciences (ICM-CSIC), 08003, Barcelona, Spain

${ }^{3}$ University of Chinese Academy of Sciences, 100049, Beijing, China

${ }^{4}$ The CAS Key Laboratory of Microwave Remote Sensing, National Space Science Center, Chinese Academy of Sciences, 100190, Beijing, China

\begin{abstract}
The sea surface winds from the CFOSAT scatterometer (CFOSCAT) are retrieved using the maximum likelihood estimator, and the inversion residual is used to sort the goodquality winds from the poor-quality ones. A twodimensional variational analysis ambiguity removal (2DVAR) scheme is then applied over the CFOSCAT swath such that a unique wind field is selected from the available local scatterometer wind vector ambiguities. The preliminary results of CFOSCAT Level 2 (L2) processing show that the retrieved wind speed is overestimated under low-wind conditions $(w<4 \mathrm{~m} / \mathrm{s})$, and is underestimated at high wind conditions $(w>15 \mathrm{~m} / \mathrm{s})$. Moreover, the inversion residual for the sweet swath (where there are more than 10 views) is generally higher than that for the nadir/outer swath. These imply that observations with different geometries (views) at the same WVC are inconsistent with respect to the geophysical model function, and thus a comprehensive calibration is highly demanded. A more detailed assessment of the CFOSCAT wind quality will be carried out after calibration and validation campaign.
\end{abstract}

Index Terms - CFOSAT, scatterometer, wind, quality control, calibration

\section{INTRODUCTION}

The China-France Oceanography Satellite (CFOSAT) was launched successfully On October, 2018. There are two scientific payloads onboard CFOSAT, i.e., the surface wave investigation and monitoring (SWIM) radar provided by the National Centre for Space Studies (CNES) of France [1], and the rotating fan-beam scatterometer developed by the National Space Science Center (NSSC) of China [2][3]. Both radar instruments are operated in Ku-band microwave frequency, and are devoted to the simultaneous observations
Table-1: Key parameters of the CFOSCAT instrument

\begin{tabular}{|c|c|}
\hline Parameter & Value \\
\hline Frequency & $13.256 \mathrm{GHz}$ \\
\hline Incidence angles & $28^{\circ}-51^{\circ}$ \\
\hline \multirow{2}{*}{ Polarizations } & Nominal (dual-pol): VV + HH \\
\hline & Test (single-pol): VV or $\mathrm{HH}$ \\
\hline \multirow[t]{3}{*}{ Antenna spinning rate } & Nominal: $3.4 \mathrm{rpm}$ \\
\hline & Low spinning rate: $3.06 \mathrm{rpm}$ \\
\hline & High spinning rate: $3.74 \mathrm{rpm}$ \\
\hline Antenna peak gain & $32 \mathrm{~dB}(\mathrm{VV}) / 31.8 \mathrm{~dB}(\mathrm{HH})$ \\
\hline Pulse duration & $1.35 \mathrm{~ms}$ \\
\hline \multirow[t]{2}{*}{ Pulse repetition rate } & Nominal (dual-pol): $75 \mathrm{~Hz}(\mathrm{VV} / \mathrm{HH})$ \\
\hline & Test (single-pol): $150 \mathrm{~Hz}(\mathrm{VV} / \mathrm{HH})$ \\
\hline Transmitted power & $120 \mathrm{~W}$ \\
\hline
\end{tabular}

of ocean surface wind vectors and directional spectra of ocean waves [4]. Currently, the CFOSAT scatterometer (CFOSCAT) together with the SWIM instrument are operated in the verification phase (i.e., the first six months after the launch), and the operational wind/wave products will be freely available to the data users in the end of April 2019.

In the nominal operation mode, CFOSCAT onboard processing generates 40 range gates (namely slices in scatterometry) with a range resolution of $\sim 10 \mathrm{~km}$. Since the surface range dimension of antenna footprint is less than 250 $\mathrm{km}$, so the effective number of slices corresponding to the footprint is actually less than 25 . The operational ground processing of CFOSCAT data is developed in China by the National Ocean Satellite Application Center (NSOAS), which consists of two essential steps. First, the Level 1 (L1) processor carries out the following procedures in sequence: geolocation of the 40 slices (before any calibration), subtraction of a noise estimate from the raw signal value of each slice, correction of potential biases in the transmitted power and the inner system loss using the internal calibration signal, and correction of systematic effects in the 
antenna gain using external calibration with transponders or Amazon rainforest. Second, at the so-called Level 2 (L2) processing, the backscatter values with similar incidence and azimuth angles are averaged in a defined swath grid (typically with resolution of $25 \mathrm{~km}$ ), and then wind retrieval is performed. The European Centre for Medium-Range Weather Forecasts (ECMWF) winds are interpolated temporally and spatially to the CFOSCAT acquisitions in order to perform wind ambiguity removal. The L2 processing generates the L2A product with averaged backscatter values and the L2B product with sea surface wind information. The produced L2A and L2B data files are both in NetCDF format, and can be converted to L2 files (including backscatter values and wind fields) in Binary Universal Form for the Representation of meteorological data (BUFR) format by a complementary processing facility.

To evaluate the wind quality derived from CFOSCAT, the innovative characteristics with the real data are overviewed in Section 2, including the observation geometry, the backscatter measurement precision, and etc. Section 3 illustrates some preliminary results from the current ground segments. Finally, conclusions and outlooks are found in Section 4.

\section{CHARACTERISTICS OF BACKSCATTER MEASUREMENTS}

The key parameters of CFOSCAT instrument are shown in Table 1. So far, the scatterometer has been operating in the nominal mode, and the other configurations will be tested soon during the rest verification phase. Consequently, we only show the results of the nominal working mode in this paper. Fig. 1 illustrates the distribution of the observation incidence and azimuth angles (the ascending pass) versus the cross-track wind vector cell (WVC) position. Each star marker represents one view that is composited from slices with similar incidence and azimuth angles. Note that the vertically-polarized (VV, red markers) geometries are slightly different from the horizontally-polarized (HH, black markers) ones, because the $\mathrm{VV}$ and $\mathrm{HH}$ beams are indeed offset by $180^{\circ}$, which is set to maximize the azimuth diversity of the observations acquired by the two different polarizations.

Fig. 2(a) illustrates the mean backscatter coefficient $\left(\sigma^{0}\right)$ of $\mathrm{VV}$ measurements (incidence $=45^{\circ}$ ) as a function of the relative observing azimuth for different ECMWF wind speeds. Here a constant correction of $3.2 \mathrm{~dB}$ is applied to the measured $\sigma^{0}$ such that the measurements (red solid curve) align well with the simulation (red dashed curve) at wind speed of $10 \mathrm{~m} / \mathrm{s}$. Note that the simulated $\sigma^{0}$ is calculated using the NSCAT-4 geophysical model function (GMF) [5]. In general, after applying a constant correction for each incidence bin, the measurements align well with the simulated $\sigma^{0}$ at medium wind speed region (not shown). However, the measured $\sigma^{0}$ is slightly lower than the simulated one at high wind conditions, and it is much larger
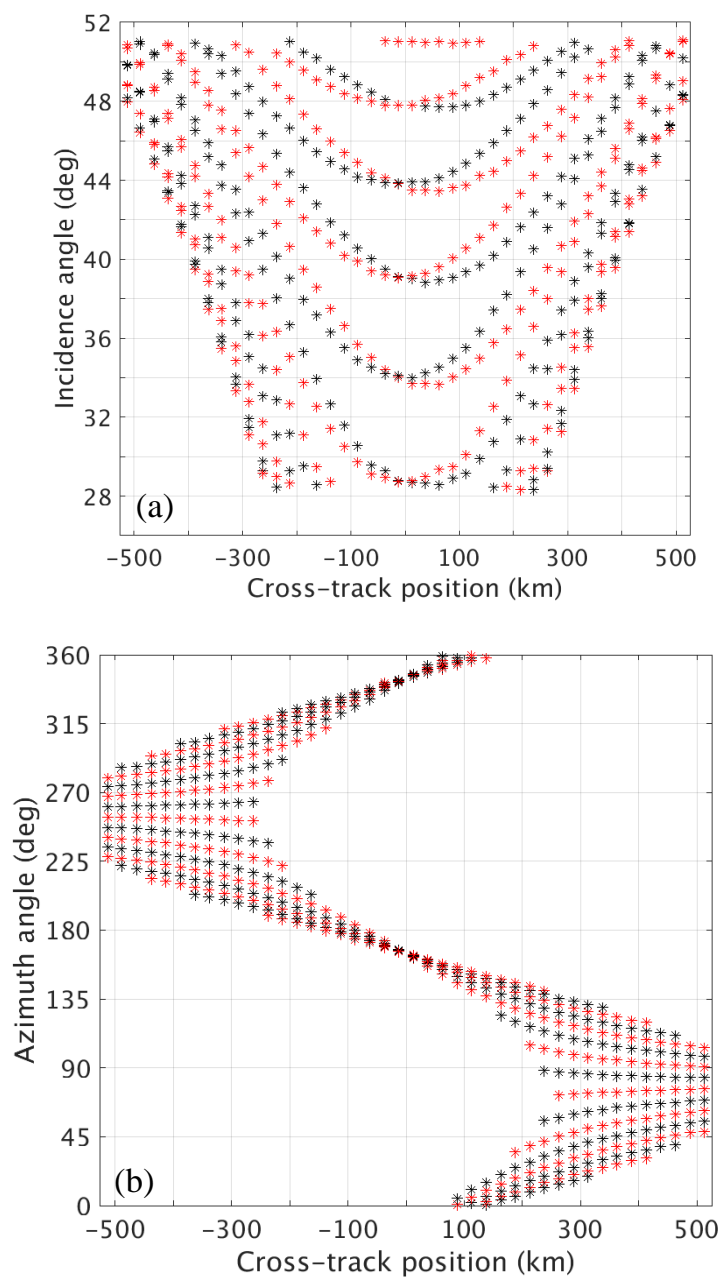

Fig. 1 The observation incidence (a) and azimuth (b) angles versus the cross-track wind vector cell (WVC) position. Black markers represent the horizontally-polarized $(\mathrm{HH})$ measurements, and red markers indicate the verticallypolarized VV measurements.

than the latter at low wind conditions. These are probably statistical artefacts due to the conditional sampling with ECMWF as reference [6]. On the other hand, the noise subtraction in the L1 processing could also affect dynamical range of the measured $\sigma^{0}$, which will be revisited in the rest verification phase. Fig. 2(b) shows the normalized standard deviation (SD) of the measured $\sigma^{0}$ for different wind speed conditions, which agrees well with the pre-launch simulation [3]. As expected, the normalized SD value at cross winds is slightly higher than that at up/down winds.

\section{WIND QUALITY}

The L2 processor is actually adapted from the Pencil-beam scatterometer Wind Processor (PenWP) developed by the Royal Netherlands Meteorological Institute (KNMI) scatterometer team in the context of European Organisation 
for the Exploitation of Meteorological Satellites (EUMETSAT) Numerical Weather Prediction (NWP)
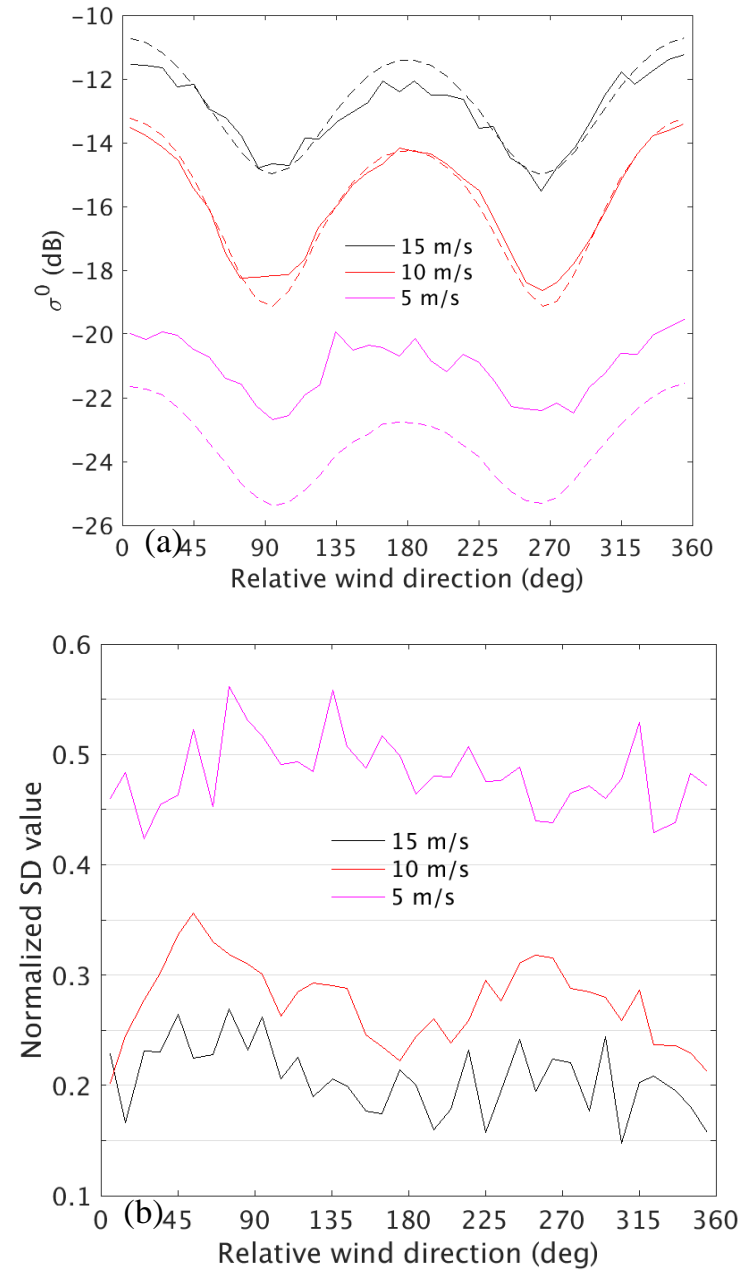

Fig. 2 (a) The mean measured backscatters of VV beam (incidence $=45^{\circ}$ ) as a function of relative observation azimuth for different wind speeds; (b) the normalized standard deviation (SD) of the measured $\sigma^{0}$ corresponding to (a). Solid curves for the measured $\sigma^{0}$, and dashed curves for the simulated $\sigma^{0}$ from NSCAT-4 GMF.

Satellite Application Facility (SAF) [7]. Basically, the CFOSCAT winds are retrieved using the MLE inversion with multiple solution scheme (MSS) [8] in combination with the two-dimensional variational analysis ambiguity removal (2DVAR) [9].

Fig. 3 illustrates the retrieved CFOSCAT wind field and the collocated ECMWF winds respectively. Generally, the scatterometer winds show similar patterns with the model wind field, particularly for the high wind regions. However, the CFOSCAT wind speed slightly lower than the model wind for high winds, and is always higher than the latter for $w<4 \mathrm{~m} / \mathrm{s}$, indicating that the backscatter characteristics in Fig. 2 is not only due to the conditional sampling. On the one hand, one should apply a more comprehensive calibration for the $\mathrm{L} 1$ processing. On the other hand, the noise subtract should be carefully examined.

The inversion residual (MLE) of the above case is
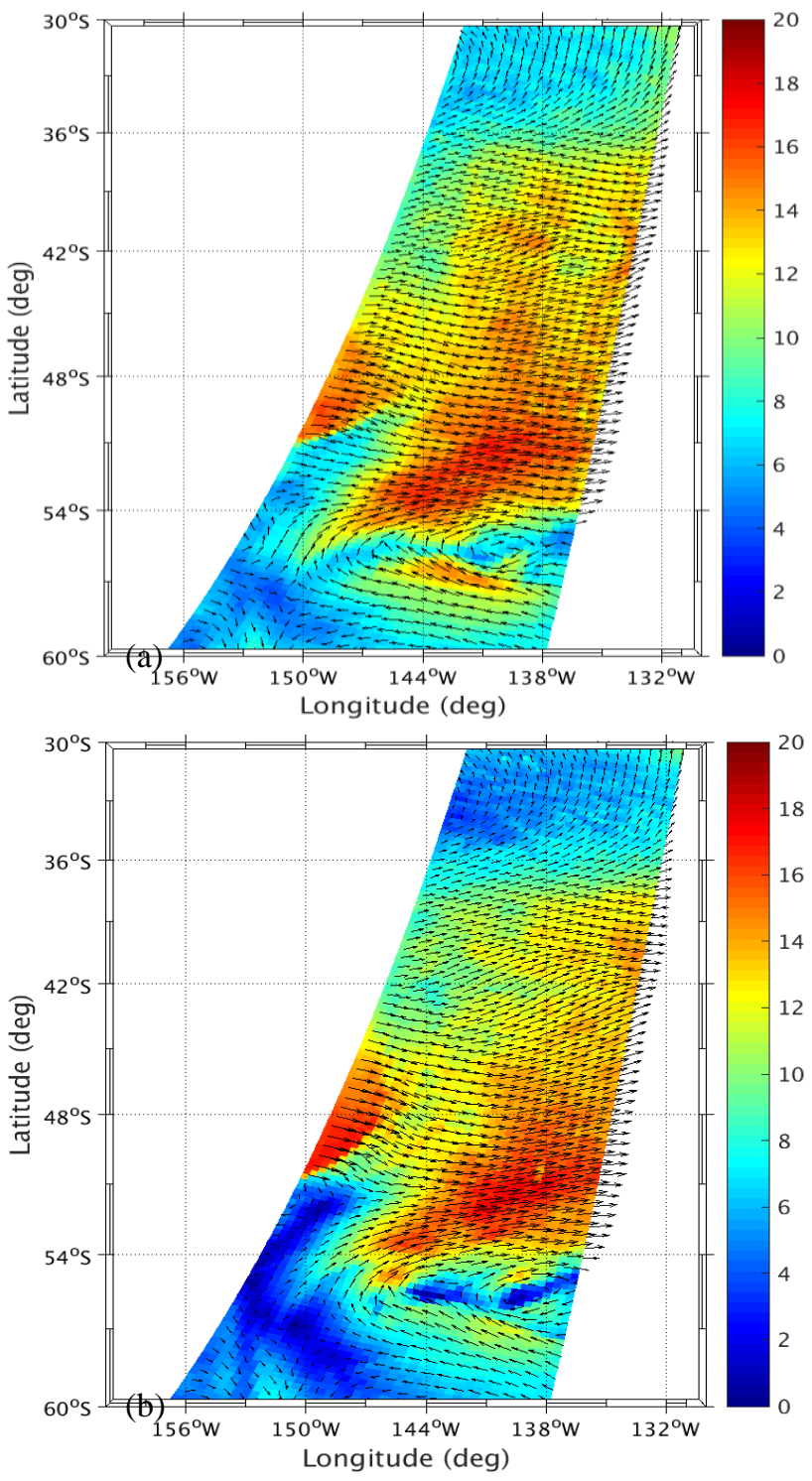

Fig. 3 (a) The retrieved CFOSCAT wind field at UTC 15:56 on December 18, 2018; (b) the collocated ECMWF forecasting winds. Colorbar indicates the wind speed.

presented in Fig. 4. Despite of the extreme large MLE values that are likely to be caused by rain and high wind variability [10], it is clear that the MLE value for the sweet swath (where there are more than 10 views, see Fig. 1) is systematically higher for that for the nadir/outer swath. Since the used L1 data is not yet well calibrated, such high MLE value is probably due to the beam inconsistency among the measurements. 


\section{CONCLUSIONS}

The recent CFOSAT scatterometer is the first spaceborne $\mathrm{Ku}$-band rotating fan-beam radar devoted to the measurements of sea surface wind vectors. This paper shows the preliminary results of the CFOSCAT ground processing.

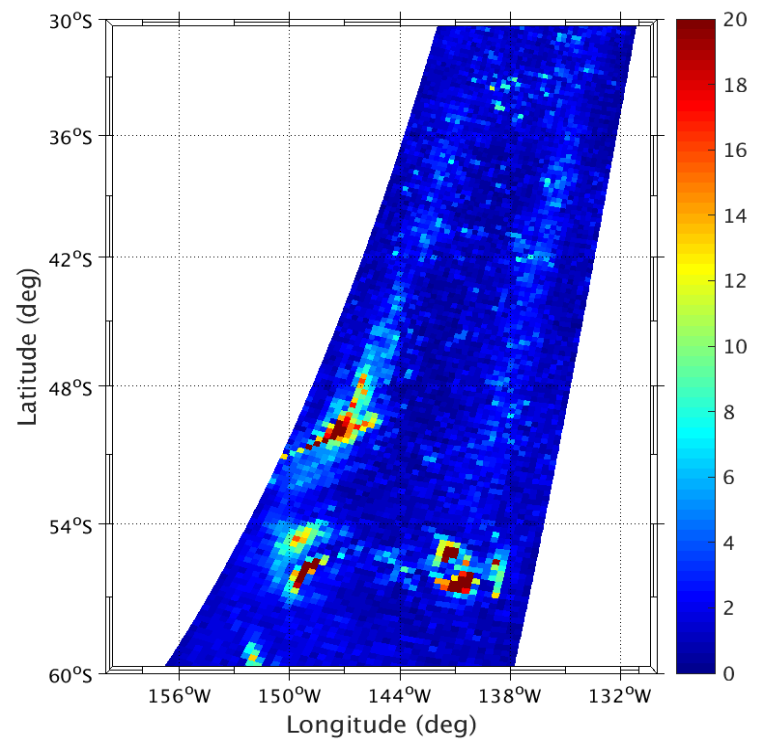

Fig. 4 The inversion residual (MLE) corresponds to Fig. 3(a)

In line with the L1 backscatter characteristics, the retrieved wind speed is largely overestimated under low-wind conditions $(w<4 \mathrm{~m} / \mathrm{s})$, and is slightly underestimated at high wind conditions $(w>15 \mathrm{~m} / \mathrm{s})$. Moreover, the inversion residual for the sweet swath is systematically higher than that for the nadir/outer swath. These imply that: first, the observations with different geometries (views) at the same WVC are inconsistent with respect to the geophysical model function, and thus a comprehensive calibration is highly demanded; second, the noise subtraction is not proper in the current L1 processing, such that the dynamic range of the measured $\sigma^{0}$ is smaller than the expectation as compared to the simulation with NSCAT-4 GMF.

When more data are available, a comprehensive assessment of CFOSCAT wind quality will be carried out using the collocated buoy winds, the HY-2B scatterometer data, and the Global Precipitation Measurement (GPM) Microwave Imager (GMI) rain data. The overall study will include two parts: first, a quality control (QC) procedure based on the wind inversion residual is developed to discern between good- and poor-quality winds. In particular, the rain impact on the CFOSCAT measurements is evaluated using the collocated GMI rain rates. Second, the quality of QCaccepted winds is assessed in terms of resolution and accuracy, using spectral analysis and triple collocation analysis with buoy winds and the European Centre for Medium-Range Weather Forecasts (ECMWF) winds. The spectral behavior and the estimated errors will be presented during the conference. These detailed results will be presented during the symposium.

\section{REFERENCES}

[1] D. Hauser, C. Tison, T. Amiot, L. Delaye, N. Corcoral, and P. Castillan, "SWIM: The first spaceborne wave scatterometer," IEEE Transactions on Geoscience and Remote Sensing, Vol. 55, no. 5, pp. 3000-3014, 2017.

[2] W. Lin and X. Dong, "Design and optimization of a rotating, range-gated fanbeam scatterometer," International Journal of Remote Sensing, 32, 8, pp. 2151-2171, 2011.

[3] W. Lin, X. Dong, M. Portabella, S. Lang, Y. He, R. Yun, Z. Wang, X. Xu, D. Zhu, and J. Liu, "A perspective on the performance of the CFOSAT rotating fanbeam scatterometer," IEEE Transactions on Geoscience and Remote Sensing, Vol. pp, no. 99, pp. 1-13, 2018. Doi: 10.1109/TGRS.2018.2858852

[4] D. Hauser, X. Dong, L. Aouf, C. Tison and P. Castillan, "Overview of the CFOSAT mission," 2016 IEEE International Geoscience and Remote Sensing Symposium (IGARSS), Beijing, 2016, pp. 5789-5792.

[5] OSI SAF, Algorithm Theoretical Basis Document for the OSI SAF wind products, SAF/OSI/CDOP2/KNMI/SCI/MA/197, and "NSCAT-4 Geophysical Model Function", 2014. Available at http://projects.knmi.nl/scatterometer/nscat_gmf/ .

[6] A. Stoffelen, "A simple method for calibration of a scatterometer over the ocean," Journal of Atmospheric and Oceanic Technology, 16(2), pp. 275-282, 1999.

[7] A. Verhoef, J. Vogelzang, J. Verspeek, and A. Stoffelen, "PenWP user manual and reference guide," NWPSAF-KN-UD009, Version 2.1, KNMI, De Bilt, the Netherlands, Feb. 2017.

[8] M. Portabella and A. Stoffelen, "A probabilistic approach for SeaWinds data assimilation," Q. J. R. Meteor. Soc., 130(596), pp: 127-159, 2004.

[9] J. Vogelzang, A. Stoffelen, A. Verhoef, J. de Vries and H. Bonekamp, "Validation of two-dimensional variational ambiguity removal on SeaWinds scatterometer data," J. Atmos. Oceanic Technol., 26, pp. 1229-1245, 2009.

[10] W. Lin, M. Portabella, A. Stoffelen, J. Vogelzang, and A. Verhoef, "ASCAT wind quality under high subcell wind variability conditions," J. Geophys. Res. Oceans, 120, pp.5804-5819, 2015. 